\title{
Comparison of experiments on bio-inspired hover kinematics with the unsteady vortex model and CFD
}

\author{
Ruben B.R. Vandenheede* \\ Delft University of Technology, Delft, Zuid-Holland, 2600AA, Netherlands \\ Luis P. Bernal†, Christian L. Morrison; Abhijit Gogulapati§, Peretz P. Friedmann, \\ and Chang-kwon Kang" \\ University of Michigan, Ann Arbor, MI, 48109, United States \\ Wei Shyy** \\ Hong Kong University of Science and Technology, Kowloon, Hong Kong
}

\begin{abstract}
In this paper we compare the force and Particle Image Velocimetry (PIV) measurements of biologically inspired hover kinematics to results of an unsteady aerodynamic vortex model (UAVM) and a Navier-Stokes (NS) solver. The baseline Reynolds number and the reduced frequency are $4.8 \times 10^{3}$ and 0.38 , respectively. We consider three versions of the hovering kinematics measured from an Agrius Convolvuli: i) without elevation angle, ii) elevation angle accounted in the pitch angle; and iii) pure sinusoidal pitch-plunge neglecting higher harmonics. The NS computations show good qualitative agreement with experiments with consistent underprediction. The time-averaged thrust coefficient obtained using NS computations are $15 \%$ to $18 \%$ of the corresponding force measurements. The standard deviation of time history of thrust coefficients, also normalized by the measured time-averaged values, is $13 \%$ to $20 \%$. The underprediction is possibly due to blockage effects in the experiments or excessive dissipation, also reflected in lower values of the vorticity compared to the PIV measurements. The UAVM captures some of the peaks in a qualitative manner. The relative difference in the time-averaged forces and standard deviation are $8 \%$ to $18 \%$ and $66 \%$ to $93 \%$, respectively. The differences in prediction of time histories are not reflected in the estimation of time-averaged forces due to cancellation effects, wherein the forces are underpredicted in the first half of the stroke and overpredicted in the second half. The discrepancies are attributed to the simplifying assumptions in the UAVM due to which the vorticity in the leading-edge vortex is overpredicted and significant differences in the wing-wake interaction are also noted.
\end{abstract}

\section{Nomenclature}

A Area swept by the wing, $\mathrm{m}^{2}$

$A R \quad$ Aspect ratio, -

$C_{L} \quad$ Lateral force coefficient, -

$C_{T} \quad$ Thrust coefficient, -

$F_{\text {lateral }}$ Lateral force, $\mathrm{N}$

$L_{\text {ref }} \quad$ Reference length

\footnotetext{
*Graduate Research Assistant, Department of Aerospace Engineering, vdh.ruben@gmail.com

${ }^{\dagger}$ Associate Professor, Department of Aerospace Engineering, lpb@umich.edu

${ }^{\ddagger}$ Graduate Research Assistant, Department of Aerospace Engineering, cmorrisz@umich.edu

$\S$ Post-Doctoral Research Fellow, Department of Aerospace Engineering, Abhijitg@umich.edu

ๆ François-Xavier Bagnoud Professor, Department of Aerospace Engineering, AIAA Fellow, peretzf@umich.edu

\|Post-Doctoral Research Fellow, Department of Aerospace Engineering, kangck@umich.edu

** Provost \& Chair Professor, Department of Mechanical Engineering, Hong Kong University of Science and Technology; also Adjunct Professor, Department of Aerospace Engineering, University of Michigan, AIAA Fellow, weishyy@ust.hk
} 


$\begin{array}{ll}M_{x} & \text { Moment around the axis x, Nm } \\ P & \text { Power, W } \\ R & \text { Semi-span, } \mathrm{m} \\ R e & \text { Reynold number } \\ T & \text { Thrust force, } \mathrm{N} \\ U_{r e f} & \text { Reference velocity, } \mathrm{m} / \mathrm{s} \\ \Phi & \text { Flapping amplitude, rad } \\ c & \text { Mean chord length, } \mathrm{m} \\ f & \text { Flapping frequency, } \mathrm{Hz} \\ h & \text { Plunge position experiment } \\ h_{0} & \text { Plunge amplitude experiment } \\ k & \text { Reduced frequency } \\ \alpha & \text { Feathering angle, rad } \\ \delta & \text { Pitch angle, rad } \\ \nu_{a} & \text { Viscosity of air } \\ \nu_{w} & \text { Viscosity of water } \\ \phi & \text { Flapping angle } \\ \rho & \text { Density } \\ \theta & \text { Elevation angle } \\ () & \text { Denotes the } 1^{\text {st }} \text { time derivative } \\ () & \text { Denotes an averaged quantity }\end{array}$

\section{Introduction}

Flapping wings have unique characteristics that can be exploited in the development of micro aerial vehicles, for example flapping wings perform very well in small spaces, they are very small and have low energy consumption. Applications can range from reconnaissance in confined spaces to search and rescue in damaged buildings. Of particular interest is the aerodynamics of small birds and insects in hover which demonstrate the agility necessary for those missions. A well-studied example of a biological flapping flyer is the hawkmoth. This moth is an excellent study object for a number of reasons. ${ }^{1}$ The wing beat of the moths is strikingly consistent. This consistent wing beat holds for an individual moth as well as comparing different moths. The main features dominant in insect flapping flight are present in the stroke of the hawkmoth, without the complications of other mechanisms present in a number of insects, e.g. clap \& fling mechanism ${ }^{2}$ and exaggerated ventral flexion.

Both numerical and experimental methods pose challenges in studying the unsteady aerodynamics of bioinspired flapping wings. As the wing size becomes smaller the flapping frequency increases and the Reynolds number decreases. Consequently, the flow fields around the flapping wings feature formation of large scale vortex structures closely related to the onset of separation and reattachment, lag between the instantaneous wing motion and the response of the flow field and the aerodynamics forces, and three dimensional effects. ${ }^{3}$ Moreover, the fluid physics associated with the flapping wing is qualitatively and quantitatively influenced by the kinematics as well as the Reynolds number. ${ }^{4}$ For fruit flies and honey bees with Reynolds number around $10^{2}$ and $1 \times 10^{3}$, issues such as turbulence are less dominant. On the other side of the low-Reynolds number regime between $1 \times 10^{4}$ and $6 \times 10^{4}$, several studies have assessed the fluid physics in a collaborative experimental and numerical framework..$^{5,6}$

In this paper experimental results for three hover kinematics derived from the hover kinematics of a hawkmoth, as presented by Vandenheede et al. $^{7}$ are compared to the results obtained with an unsteady vortex model ${ }^{8}$ and a high fidelity computational fluid dynamics (CFD) code, ${ }^{6}$ solving the Navier-Stokes Equations. The Reynolds number and reduced frequency for the present results are representative of a hawkmoth in hover, i.e. $R e=4.8 \times 10^{3}$ and $k=0.38$ respectively. A pitch-plunge apparatus is used to produce the hover kinematics. Although this is a significant change compared to actual flapping wing kinematics, this simplification enabled the high resolution force and velocity measurements necessary to validate the results. We primarily focus on both the time-averaged values and the time histories of the thrust generation and the corresponding vorticity field. The time-averaged values are relevant for micro aerial vehicles, when the flapping timescale is orders of magnitude smaller than the vehicle time scale. 
Moreover, the instantaneous force histories are essential for flight dynamics modeling and control.

An intriguing feature of biological flyers is the flexibility observed in their wings. Recent review by Shyy et al. ${ }^{9}$ experimental study by Ramananarivo, Godoy-Diana, and Thiria, ${ }^{10}$ and numerical study by Kang et al. ${ }^{11}$ have suggested that wing flexibility can enhance the aerodynamic performance by tuning its kinematics to its wing material properties. In this paper we use a rigid wing to simplify experimental measurements and focus on the differences and similarities in the results obtained using experimental and numerical methods. Discussion on the effects of flexibility on the aerodynamic performance can be found in the abovementioned studies.

The outline is as follows. We first describe the case setup, followed by a description of the experimental and numerical methods in Section II. The comparisons of the force coefficients and the flow structures are shown and discussed in Section III. Finally, the conclusions are presented in Section IV.

\section{Methodologies}

\section{A. Scaling and Non-dimensional Parameters}

Several hover kinematics, inspired by the kinematics of a hawkmoth (Agrius Convolvuli), are used in this study. In this section we describe the process used to derive the pitch-plunge kinematics from the hawkmoth hover kinematics. As a starting point for determining the relevant scaling and non-dimensional parameters, the values for the Agrius Convolvuli wing motion are presented first followed by the corresponding values for the pitch-plunge kinematics.

\section{Hawkmoth, Agrius Convolvuli}

The Agrius Convolvuli is a subspecies of the hawkmoth, Sphingidae. It is a large species with a wingspan of about $80-105 \mathrm{~mm}$. Similarly to hummingbirds, they have evolved to feed on nectar while hovering in front of a flower. This capability makes them an excellent study object for flapping hovering flight. The kinematics of this moth in hovering flight have been measured by Ellington, ${ }^{12}$ cf. Section B. The motion is relatively simple and does not include a clap and fling mechanism. The Agrius Convolvuli is four-winged, but the motion of the wings on both sides in hover is nearly the same and can be assumed to act as one wing. The wing planform of both wings together is similar to the Zimmerman planform and will be modeled as such.

Table 1: Agrius Convolvuli parameters in hover and experiment parameters

\begin{tabular}{l|l|l}
\hline Parameter & $\begin{array}{l}\text { Value Agrius } \\
\text { convolvuli }\end{array}$ & $\begin{array}{l}\text { Value Exper- } \\
\text { iment }\end{array}$ \\
\hline Mean chord length $c$ & $18.3 \mathrm{~mm}$ & $62.4 \mathrm{~mm}$ \\
Semi-Span $R$ & $50.5 \mathrm{~mm}$ & $241.3 \mathrm{~mm}$ \\
Aspect ratio $A R$ & 2.76 & 3.87 \\
Flapping frequency & $26.1 \mathrm{~Hz}$ & $0.15 \mathrm{~Hz}$ \\
Flapping/Plunging & $2 \mathrm{rad}$ & $129.1 \mathrm{~mm}$ \\
amplitude $\Phi / \mathrm{h}$ & & \\
\hline \hline
\end{tabular}

The parameters determining the dynamics are presented in Table 1. The flapping frequency and amplitude are those of the moth in hover. In order to calculate the relevant dimensionless parameters for hover, a reference point on the wing needs to be chosen. In general the wing tip is used for this purpose, ${ }^{9}$ however since the objective is to capture the most relevant phenomena and try to represent these in a pitch-plunge motion, the tip velocity will make the scaling parameters too large. A reasonable approximation is to use three quarters of the span $\left(L_{r e f}=0.75 R_{m}\right)$.

$$
U_{\text {ref }}=2 \Phi f L_{r e f}
$$

The Reynolds number can then be rewritten as follows. 


$$
R e=\frac{U_{r e f} c_{m}}{\nu_{a}}=\frac{2 \Phi f_{m} L_{r e f} c_{m}}{\nu_{a}}
$$

In turn the reduced frequency, equation 3 , is based on the same reference velocity.

$$
k=\frac{\pi f_{m} c_{m}}{U_{r e f}}
$$

With the reference velocity based on three quarters of the span the Reynolds number and reduced frequency are $4.8 \times 10^{3}$ and 0.38 respectively.

\section{Relevant Non-dimensional Parameters}

We cosider a pitch-plunge motion, hence the reference velocity is equal to the average velocity during half of the period which is given in equation 4 and depends only on the amplitude and frequency of the motion.

$$
U_{\text {ref }}=4 f h_{0}
$$

Substitution of the reference velocity, equation 4, leads to the following expression for the reduced frequency of the experiment. Note that in the pitch plunge case, for a certain wing, the reduced frequency is solely dependent on the amplitude of the motion.

$$
k=\frac{\pi c}{4} \frac{1}{h_{0}}
$$

Similarly the Reynolds number can be written as

$$
R e=\frac{4 c}{\nu_{w}} f h_{0}
$$

In these sets of kinematics, the Reynolds number is kept constant at the value of the hawkmoth, i.e. $4.8 \times 10^{3}$. The reduced frequency is kept at 0.38 .

\section{B. Hover kinematics}

The kinematics used in this study are based on the kinematics of the hawkmoth. The rotation axis of the pitch motion coincides with the leading edge at the root of the airfoil. Three motions are considered in this study, the first hover motion ignores the elevation angle of the hawkmoth kinematics and simply converts the flapping and feathering angle, as shown in Figure 1a. This motion will be referred to as hover motion 1 or HM1. The second hover motion, Figure 1b, incorporates a correction in the flapping and feathering angle for variations in elevation angle. This motion will be referred to as hover motion 2 or HM2. The third motion is a pure sinusoidal motion with the same pitch and plunge amplitudes as the basic Agrius Convolvuli kinematics. The sinusoidal kinematics are plotted in Figure 1c.

\section{Experimental Setup}

\section{Water Channel and Wing Planform}

The experiments are performed in The University of Michigan water channel. The channel has total volume of about $19 \mathrm{~m}^{3}$. The test section measures 610 by $610 \mathrm{~mm}$ and is $2.44 \mathrm{~m}$ long. The transparent test section walls facilitate flow visualization and particle image velocimetry experiments. The channel is capable of producing very low turbulence flow, the free turbulence intensity is less than $1 \%$. Flow speeds can range from 5 to $40 \mathrm{~cm} / \mathrm{s}$. For this experiment there is no flow in the channel, as we are investigating a hover case.

A Zimmerman wing is used in all experiments; the wing has a chord of $79.4 \mathrm{~mm}$ and a span of $241.3 \mathrm{~mm}$. The planform shape consists of two ellipses with the major axis equal to the span and the midpoint of the ellipse is the quarter chord position, cf. Figure 2a. The wing planform is laser-cut (PLS6.75, Universal Laser Systems) out of a transparant acrylic plate with a thickness of $2.75 \mathrm{~mm}$ and the wing edges are sharp right angles. The thickness of the wing is substantial and the wing is henceforth assumed to be rigid; during the experiments no deformation of the wing was observed. The wing is clamped and mounted on a rig with two motors, hanging vertically in the water channel, as shown in Figure 2b. The motors are capable of 


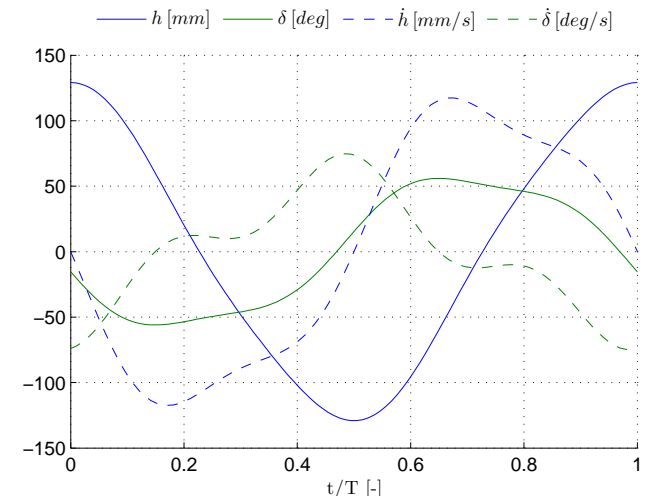

(a) Pitch-plunge hover motion experiment ignoring the loss of elevation angle (hover motion 1)

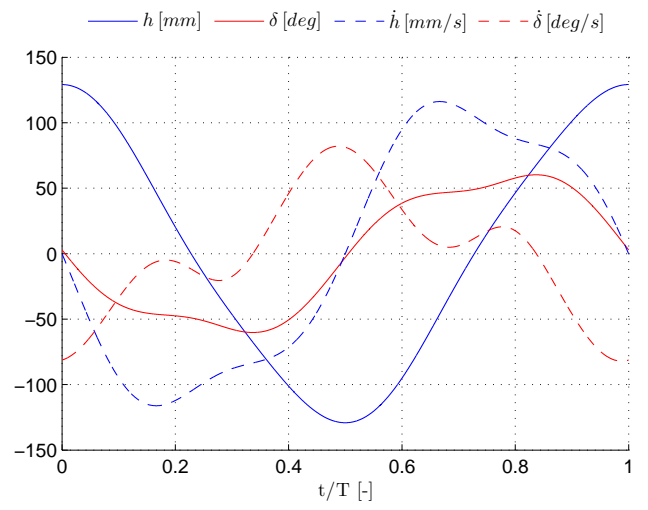

(b) Pitch-plunge hover motion experiment with compensation for the loss of elevation angle (hover motion 2)

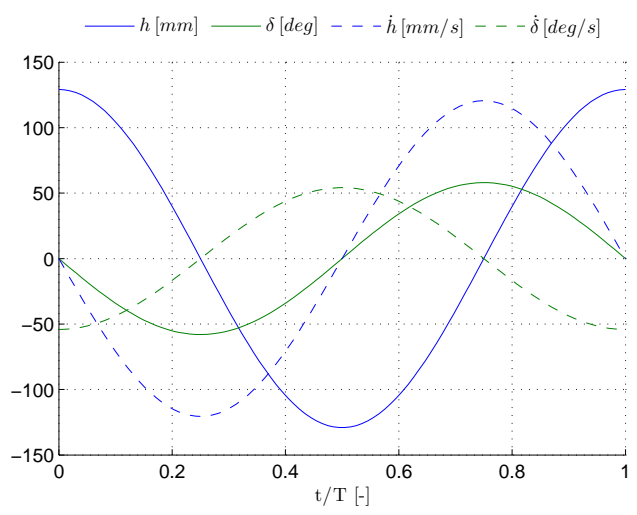

(c) Pure Sinusoidal pitch-plunge motion with the same amplitude and frequency as the Agrius Convolvuli

Figure 1: Resulting kinematics pitch-plunge kinematics of the experiments

performing pitch-plunge motions with a high degree of accuracy.

\section{Force Data Acquisition}

The force data is acquired using the ATI Mini 40 force/torque sensor mounted on top of the wing clamp. The raw sensor data is then calibrated and filtered using Butterworth low-pass filter to reduce the noise.

Per motion, force data is acquired in 6 separate sets of 25 cycles. The first five cycles are cropped from each measurement eliminating any start up phenomena that might occur. The data is then calibrated and converted to thrust and lateral force components. The thrust is defined as the force perpendicular to the plunge motion, similarly the lateral force is the force lateral to the plunge motion.

The thrust and lateral force components are filtered with a low pass Butterworth filter with a cut off frequency of $3 \mathrm{~Hz}$. To eliminate the phase shift introduced by the filter, it is run over the data in the forward and reverse time direction using MATLAB 's filtfilt function. This process ensures zero phase distortion and squares the magnitude of the original filter transfer function.

Next the remaining 120 cycles are phase averaged and the sample mean and the corresponding standard deviation are calculated. 


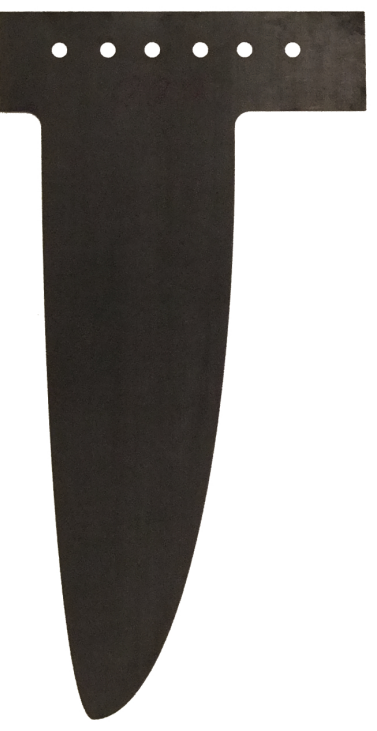

(a) Wing planform

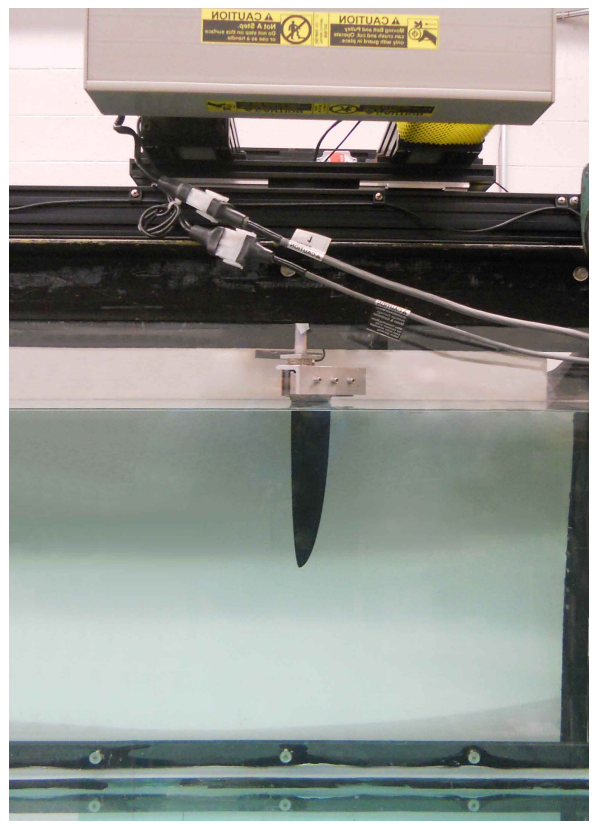

(b) Wing vertically mounted in the channel

Figure 2: Experimental Setup

\section{Particle Image Velocimetry}

The Particle Image Velocimetry (PIV) setup consists of a pulsed Nd:YAG laser (Spectra-Physics PIV-300), an optical setup to form the laser sheet, an external timing system (a BNC 555 pulse delay generator and the Stanford Research Systems' DG535) a high resolution 14bit cooled CCD camera system (Cooke, PCO4000) with a Nikon Micro-NIKKOR $105 \mathrm{~mm}$ lens. The camera has a sensor of 4008 by 2672 pixels. The flow is seeded with Titanium(IV) oxide, rutile powder.

In setup the laser sheet is horizontal, parallel to the water surface, and the camera is underneath looking up, cf. Figure 3. The plunge amplitude is about $130 \mathrm{~mm}$, to obtain the required spatial resolution three camera positions are used and the data is stitched together during post-processing. The camera is mounted on a slide and shifted to 3 positions that have an overlap region. The stitching procedure is completely discrete in order to make sure that discrepancies between images would be spotted visually. The camera lens is focused to produce a magnification of approximately 14 pixels per $\mathrm{mm}$. Using this technique a field of view of 6225 by 3525 pixels (or 437 by $247 \mathrm{~mm}$ ) is obtained, capturing the entire plunge motion.

\section{Unsteady Aerodynamic Vortex Model}

The unsteady aerodynamic model is based on two-dimensional potential flow and uses a vorticity/circulation approach to compute the aerodynamic loads. The formulation was originally derived for rigid wings in hover. ${ }^{13,14}$ The model has been subsequently modified to account for spanwise and chordwise wing flexibility $^{15,16}$ and freestream due to forward flight speed. ${ }^{8}$ The effect of the Reynolds number is also incorporated into the calculation of shed vorticity. ${ }^{8}$

Transient and time-averaged forces, computed using the approximate model, were compared with those obtained from CFD-based computations for rigid airfoils as well as wings in hover and forward flight. ${ }^{15,16,8}$ For all cases considered, the approximate unsteady aerodynamic loads show reasonable agreement with CFD-based results.

The overall approach is as follows. First, the wing is divided into several spanwise stations, where each section is represented as an airfoil. For each airfoil, an airfoil-wake surface that captures the airfoil degrees of freedom (DOF), and approximates the geometry of the shed wake, is identified. Next, the airfoil and the airfoil-wake surface are transformed to a circle in the complex plane using a conformal mapping. Thus, the 


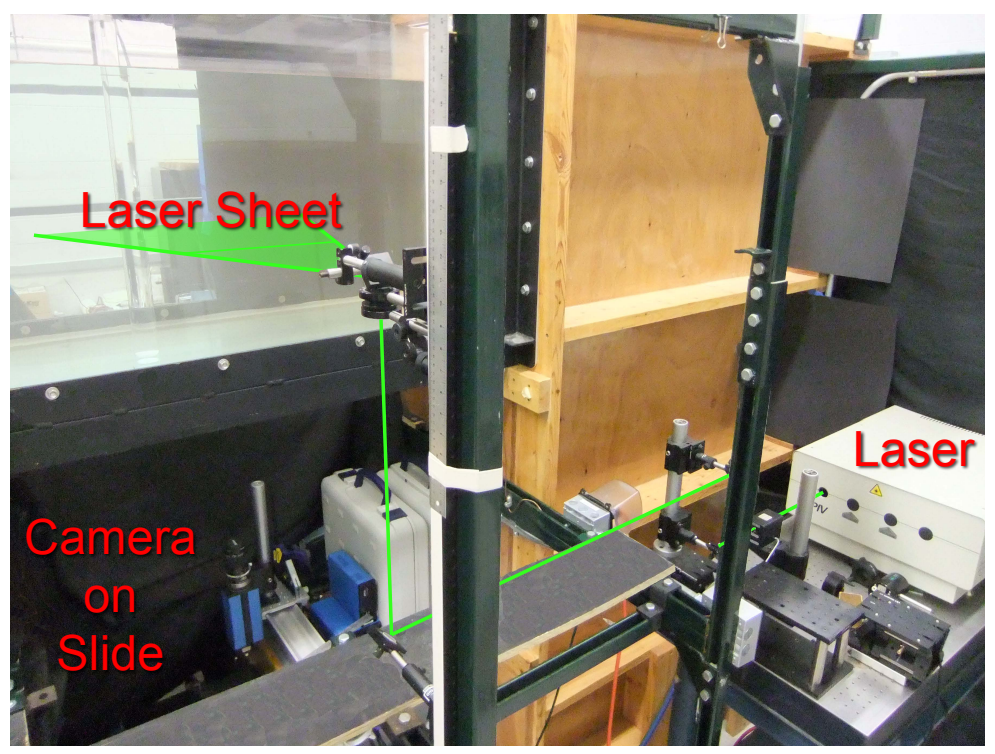

Figure 3: Representation of the PIV setup parallel to the water surface

airfoil-bound and shed wake vorticity are computed on the complex plane. The quasi-steady component of vorticity is obtained by neglecting the effect of the shed wake. The strength of shed vorticity is computed by enforcing a stagnation condition at the leading edge (LE) and a Kutta condition at the trailing edge (TE). The airfoil bound vorticity is obtained as a sum of the quasi-steady and wake-induced vorticity on the airfoil. Next, the vorticity in the complex plane is transformed back to the airfoil-wake surface (physical plane) using an inverse transform. The unsteady loads acting on the airfoil are obtained from the total vorticity using the unsteady Bernoulli equation. Finally, the shed vorticity is convected using the Rott-Birkhoff equation, which is derived from Biot-Savart law for two dimensional flow.

For the case of hover, the vortices that are shed during previous cycles remain in the vicinity of the wing and therefore influence the forces generated by the wing even after several subsequent cycles. Consequently, the mean forces were computed once the forces reached an approximate steady state. For all the cases considered, an approximate steady state was reached after the second cycle.

Simulations also showed that decreasing the vortex core improved correlation with experimental results. Decreasing the vortex core size promotes vortex roll up. Thus it appears that a core size of $0.00625 c$ captures the vortex dynamics most accurately. Furthermore, decreasing the size of the vortex core also decreased the noise generated due to interaction of the vortices with the airfoil thereby improving quality of the solution. It appears that decreasing the core size further had a comparatively minor impact on the forces (cf. Figure 4).

\section{E. Computational Fluid Dynamics: Navier-Stokes Equation Solver}

The governing equations for fluid motion given by the Navier-Stokes equations are solved with Loci-STREAM, ${ }^{4,6}$ which is a three-dimensional, unstructured, pressure-based finite volume solver written in the Loci-framework. It employs implicit first or second order time stepping and treats the convection terms using the second order upwind-type scheme and the pressure and viscous terms using second-order schemes. The system of equations resulting from the linearized momentum equations are handled with the symmetric Gauss-Seidel solver. The pressure correction equation is solved with either the GMRES linear solver with Jacobi pre-conditioner provided by PETSc ${ }^{17,18,19}$ or the BoomerAMG ${ }^{20}$ linear solver provided by hypre. The Loci-framework is by design rule-based highly parallelizable framework for finite volume methods. ${ }^{21}$ The geometric conservation law, ${ }^{22}$ a necessary consideration in domains with moving boundaries, is satisfied. ${ }^{23}$ The motion of the wing is realized by rigidly moving the computational mesh., ${ }^{4,6}$

The computational grid to solve the Navier-Stokes equations consists of mixed brick and tetrahedral cells around the Zimmerman wing, see Figure 5. To assess the grid size sensitivity four grids with different spatial resolutions are utilized with $0.34 \times 10^{6}$ (blue solid), $0.51 \times 10^{6}$ (black dashed-dotted), $0.73 \times 10^{6}$ (red 


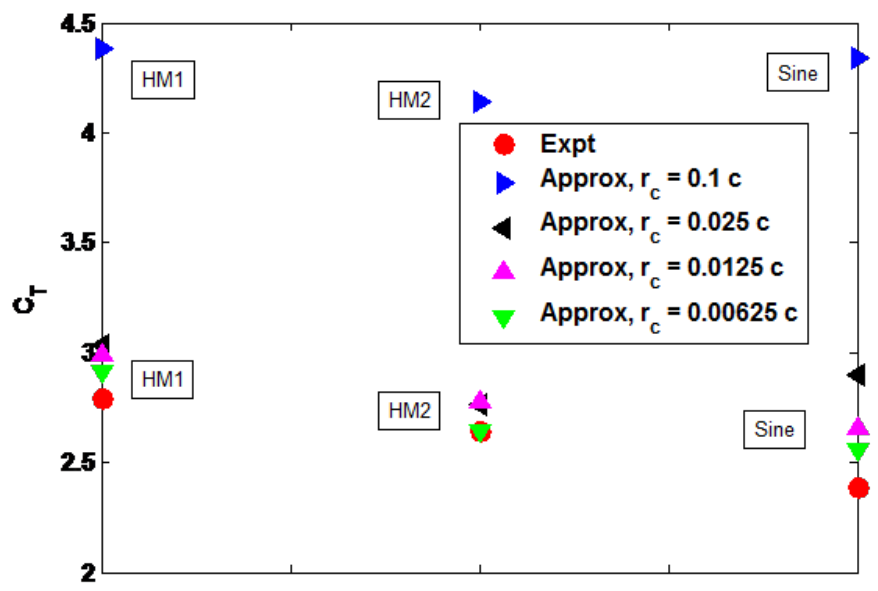

Figure 4: Comparison of the force coefficients for different core radii

dashed), and $5.7 \times 10^{6}$ (magenta dotted) cells for each mesh. For the time step sensitivity analysis 250 (blue solid), 500 (red dashed), and 1000 (black dotted) time steps per motion cycle were chosen on the grid with $0.51 \times 10^{6}$ cells. From the results shown in Figure 6, the intermediate grid with $0.51 \times 10^{6}$ cells and 500 time steps per motion cycle show solutions with sufficient spatial and temporal resolution. The thrust on the finest mesh $\left(5.7 \times 10^{6}\right)$ has the highest peak by around $7, \%$ but the chosen mesh is able to capture the physics closely. The fluid flow is assumed to be laminar. The first grid spacing from the wing surface is set to $2.5 \times 10^{-3}$ and the outer boundary of the computational grid is located at 30 chords away from the wing. At the outer computational boundary zero velocity and reference density are assigned. On the wing surface the no-slip condition is applied.

\section{Results and Discussion}

\section{A. Non-dimensionalization of the Data}

The force data is non-dimensionalized by the dynamic pressure (based on the reference velocity $U_{\text {ref }}$ equal to $4 f h_{0}$, Section A 2) and the projected wing surface area. The thrust coefficient is then defined as shown in equation 7.

$$
C_{T}=\frac{T}{q S}
$$

Similarly the lateral force coefficient is normalized by the same dynamic pressure and the projected wing surface area.

\section{B. Comparison of Force Generation}

The force coefficients, as described in Section A, for the three motions are presented in this section. The first two cycles were cropped from the data set to remove the initial transient effects and the remaining 3 cycles were phase averaged for forces obtained from the NS computations and the unsteady aerodynamic vortex model. The comparison of the time history of the forces is shown in Figure 7. The unsteady aerodynamic vortex model took approximately six hours on 12 CPUs to compute for five motion cycles, while the NavierStokes computations took approximately 16 hours on 24 Intel Xeon CPUs.

The shape of the time history of the force coefficients for the NS calculations is similar to the measurements for all motions with slight under-prediction. As with the experiments there is a slight asymmetry in the force coefficients for the equivalent of the up and downstroke. For HM1 the NS results can almost be linearly scaled to fit the experimental results. There is a small difference for HM2 in each stroke, that is the disproportion between the first and second maximum in the thrust force coefficient is slightly more pronounced in the NS 


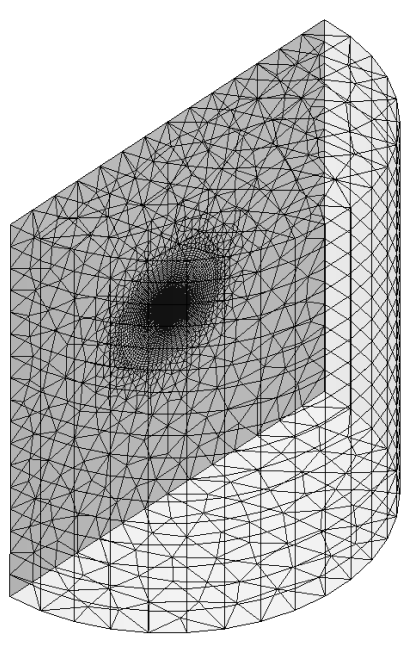

(a) Computational domain

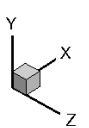

Figure 5: Computational grids for the Zimmerman wing
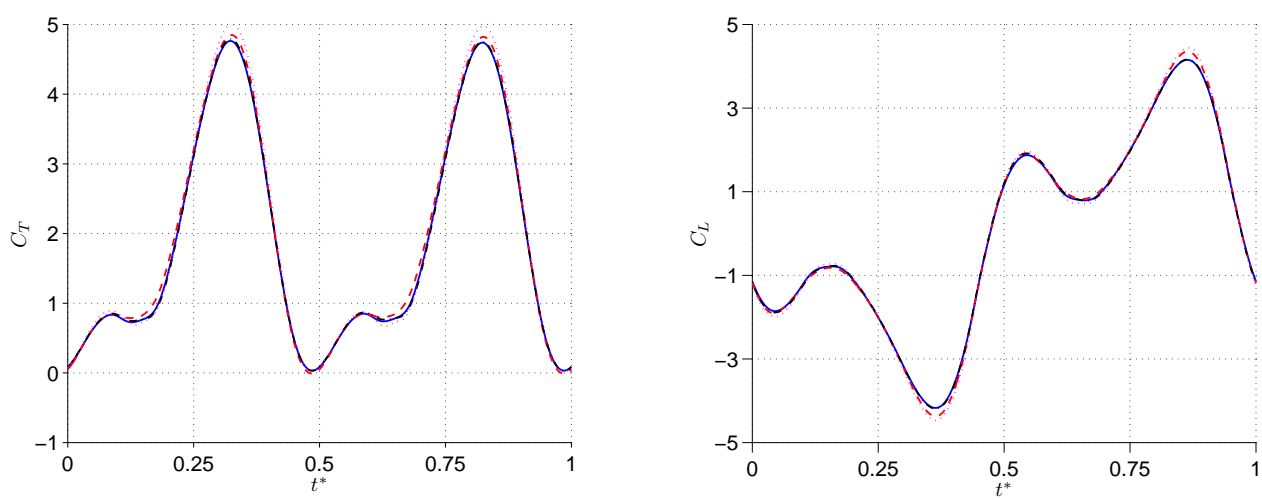

(a) Spatial
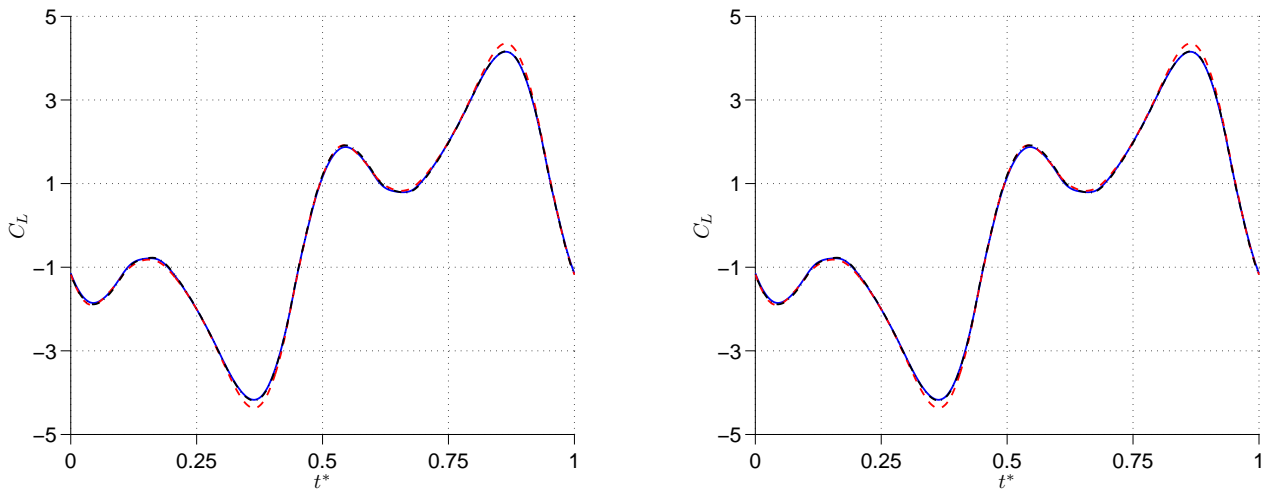

(b) Temporal

Figure 6: Spatial and temporal sensitivity tests for the pure sinusoidal motion. 


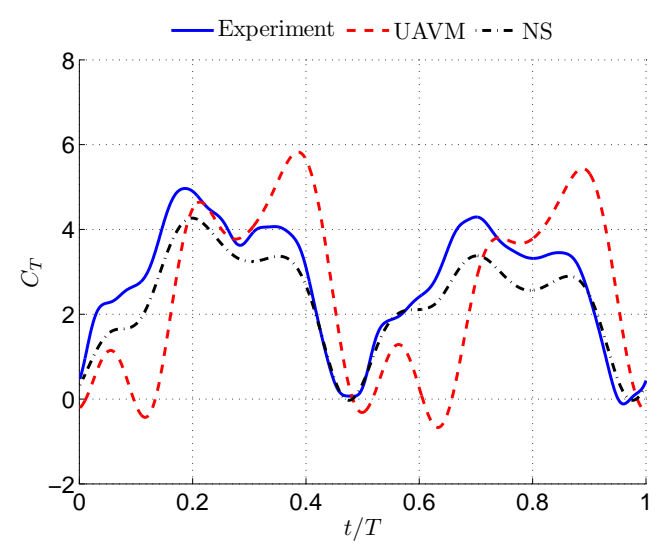

(a) Thrust coefficient hover motion 1

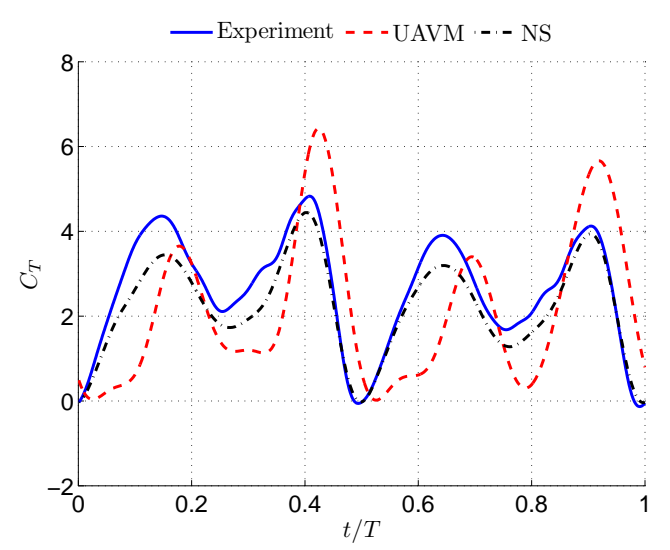

(c) Thrust coefficient hover motion 2

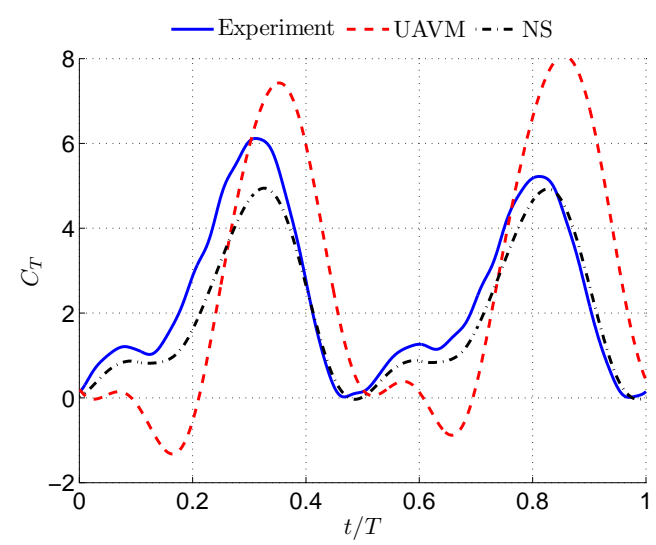

(e) Thrust coefficient pure sinusoidal motion

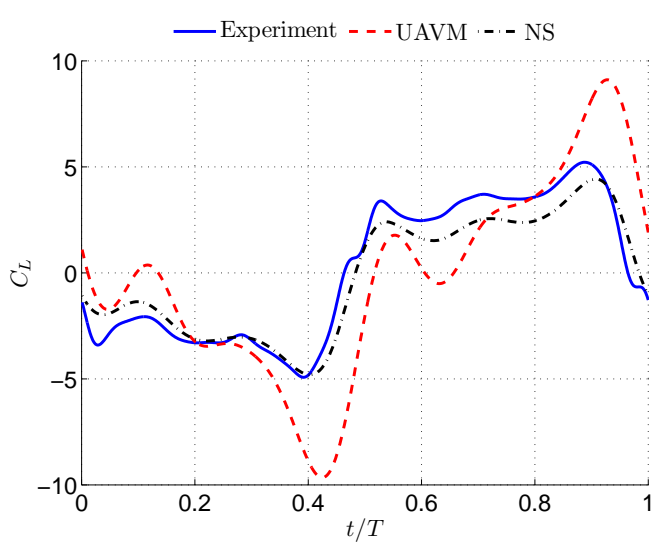

(b) Lateral force coefficient hover motion 1

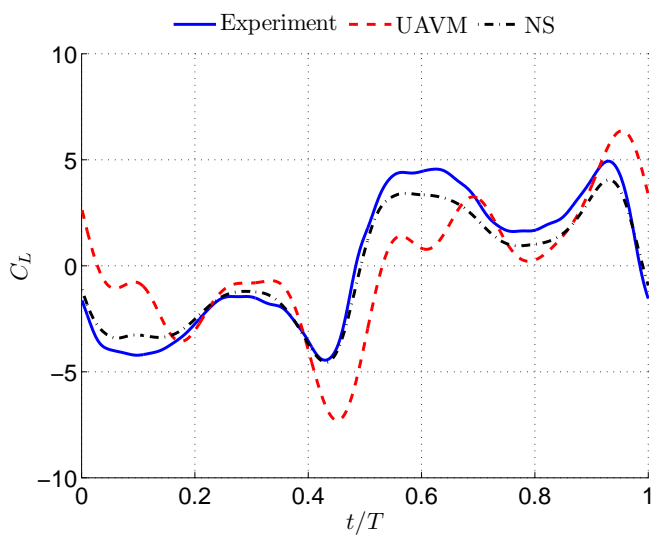

(d) Lateral force coefficient hover motion 2

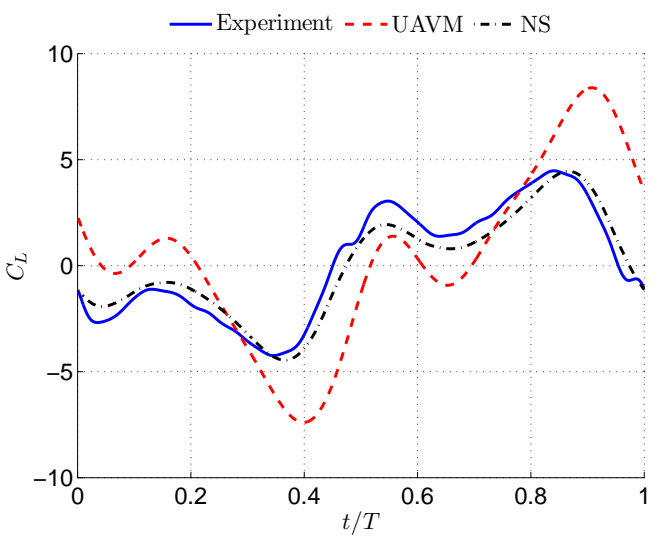

(f) Lateral force coefficient pure sinusoidal motion

Figure 7: Comparison of the force coefficients of all three motions for the experiment, unsteady aerodynamic vortex model and the NS computations 
calculations. And the difference between the up and downstroke are less pronounced for the purely sinusoidal motion. For all motions however the shape is very similar. The time at which peaks and valleys occur in the force coefficients is nearly the same, the maximum lag between two maxima is $1.8 \%$ percent of the period (occuring in HM1).

The unsteady aerodynamic model predicts a shape of the time history of the force coefficients that is less similar to the experiments. The unsteady aerodynamic vortex model under-predicts the force coefficients of the experiment in the beginning of the stroke, $t^{*}=0-0.2$ and over-predicts the force coefficients towards the end of the stroke, generally for $t^{*}=0.3-0.5$; and similarly for the reverse stroke. This trend is observed for all three motions. The location of the peaks is accurately predicted by the model, the maximum lag between two maxima is $5.1 \%$ of the period and occurs in HM2.

The average force coefficients for the experiment, the NS calculations, and unsteady aerodynamic vortex model are summarized in Table 2. The forces coefficient computed and measured are of the same order of magnitude and the differences are always smaller than $31 \%$ with respect to the experiment.

Table 2: Base motion average force coefficients and standard deviation, experiment (Exp) versus NS (averaged over the last 4 cycles) and UAVM (averaged over the last 4 cycles)

\begin{tabular}{c|ccc|ccc|ccc}
\hline & \multicolumn{3}{|c|}{ HM1 } & \multicolumn{3}{c|}{ HM2 } & \multicolumn{3}{c}{ Sine } \\
& Exp & NS & UAVM & Exp & NS & UAVM & Exp & NS & UAVM \\
\hline$C_{T}$ & 2.79 & 2.31 & 2.54 & 2.64 & 2.23 & 2.17 & 2.39 & 1.97 & 2.57 \\
Mean $\Delta C_{T}$ & - & -0.475 & -0.243 & - & -0.41 & -0.479 & - & -0.421 & 0.184 \\
Std $C_{T}$ & - & 0.379 & $1.84\left(1.25^{\mathrm{a}}\right)$ & - & 0.295 & $1.56\left(1.01^{\mathrm{a}}\right)$ & - & 0.499 & $2.21\left(1.55^{\mathrm{a}}\right)$ \\
\hline \hline
\end{tabular}

On average the forces computed with NS are between 82 and $87 \%$ of the coefficients measured in the experiment; the NS computations underpredict the force coefficients consistently as shown in the time histories in Figure 7. The possible reasons for these differences may be found in the boundary conditions of each setup. First, the experimental setup consists of water channel walls near wing motion, leading to blockage effects, while in the NS computations the outer boundary of the computations domain is located orders of magnitude away from the motion. Blockage effects would increase the forces measured. Second, in the experiments an offset between the center of the motions and the center of the water channel could cause an asymmetry in the measured forces during the up and downstroke. The flow in the NS computation is assumed to be fully laminar without an attempt to resolve small turbulence scales to capture turbulence or laminar-to-turbulence transition. These potential sources of disagreement between the experiment and CFD have not been quantified and are being investigated.

Similar to predicting the shape of the time history of the forces, the prediction of the average force coefficients by the unsteady aerodynamic model is also less accurate. The difference in averaged force coefficients for HM1 and HM2 is about $20 \%$, about $7.5 \%$ for the thrust coefficient of the pure sinusoidal motion, and $31 \%$ for the lateral force coefficient. These time-averaged thrust coefficients are closer to the experimental values compared to the NS computations, due to cancellation effects with an underprediction in the first part of the stroke, $t^{*}=0.05-0.25$ and overprediction of the forces in the second part of the stroke, $t^{*}=0.3-0.5$. Potential sources of these discrepancies are discussed in Section C. The standard deviation $S t d C_{T}$ for the unsteady aerodynamic vortex model is indeed much greater than for the NS computations as shown in Table 2. Note that $S t d C_{T}$ reduces when the time history of thrust coefficient is corrected for the phase difference.

The flow visualization data ${ }^{24}$ and the flow field obtained from the NS computations revealed the presence of tip vortices and a spanwise component of the flow from the tip towards the root of the wing. Compared to the tip vortices on a hovering flat plate at lower Reynolds numbers, i.e. $R e=100$, the size of the tip vortices observed in this study was smaller, also due to the narrower wing shape near the tip. On the other hand, Shyy and $\mathrm{Liu}^{25}$ investigated the role of LEVs and spanwise effects at Re ranging from 10 to 6000 for insect-like wings and kinematics. They observed that the spanwise effects were the greatest at $R e=6000$ for hawkmoth kinematics and the spanwise flow became weaker as the Reynolds number is lowered. More detailed and concrete study of the three-dimensional spanwise effects as a function of $R e, k$, and kinematics is left as future study.

\footnotetext{
${ }^{\text {a }}$ Phase lag corrected std
} 


\section{Comparison of Vorticity Field}

Complementary to the force data acquisition, the vorticity fields were measured and computed. To attain the vorticity field in the experiment two dimensional particle image velocimetry is performed. From the experiments it was found that the flow topology at half span is representative for the flow topology on the majority of the airfoil. ${ }^{24}$

The vorticity field reveals a number of large-scale features, e.g. formation of a leading edge vortex. These large-scale features are observed by all three methods and differ distinctly for all three motions.

Figure 8 shows the vorticity of the phase-averaged flow field for HM1 at half span and phases as indicated for the experiment, the NS computations, and the unsteady aerodynamic vortex model. At the beginning of the stroke we see that the leading edge vortex (LEV) from the previous half stroke interacts with the wing and breaks into two pieces, a small part flowing over the leading edge dissipating almost immediately and a larger part moves along the airfoil surface to the trailing edge where it interacts with the trailing edge vortex (TEV) or starting vortex. There is evidence of one LEV forming from approximately $t^{*}=0.2$ until the end of the stroke. The largest thrust coefficient occurs at $t^{*}=0.2$. After the change in pitch rate at $t^{*}=0.4$, where the largest force coefficient occurs, another starting vortex appears at the trailing edge. This vortex and the starting vortex at stroke reversal, which has opposite circulation, combine to form a persistent vortex structure.

The phase-averaged vorticity for HM2 is plotted in Figure 9. Similar to HM1, the LEV interacts with the wing, right after the stroke reversal. The LEV breaks into two parts, where the larger part joins the starting vortex at the trailing edge. The other part remains around the leading edge and is entrained and annihilated by the new LEV having opposite circulation. In this case the LEV detaches between $t^{*}=0.2$ and 0.4, stimulated by the increase of the pitch angle at $t^{*}=0.25$. A new LEV forms around $t^{*}=0.4$. These flow features correlate closely with the two peaks in force coefficients. The first peak is called the wake-capture peak, ${ }^{26}$ where the wing-wake interaction results in thrust enhancement. The second thrust peak is mainly due to the generation of the LEV on the suction side of the wing. ${ }^{26}$

In Figure 10, the phase-averaged vorticity and streamlines for the sine motion are plotted. At the start of the stroke during stroke reversal, the vorticity contours show significantly weaker LE and TE vortices compared to the other two kinematics, which is consistent with the relative low force coefficient measured at these phases. For this kinematics the LEV is observed first on the image corresponding to $t^{*}=0.2$. The force coefficients at the beginning of the stroke are small but as the LEV grows larger the force coefficients surmount the maximum coefficients of both HM1 and HM2. There is no formation of a TEV at stroke reversal, in contrast to HM1 and HM2.

The NS computations of the vorticity field compares well to the experiment. The largest difference occurs at $t^{*}=0.1$, at this phase there is a negative vorticity region on the bottom of the airfoil that is not present in the PIV measurements. The maximum positive vorticity in that same region is roughly twice as large as the experiment. For all other phases the vorticity field in the near field, within 1 chord length of the airfoil, is nearly identical to the measurements; further away from the airfoil the NS computations displays a larger diffusion of the vorticity compared to the experiment, which may be due to the numerical viscosity or the presence of the walls in the experiments.

The vorticity flow field for the UAVM is obtained first by calculating the induced velocity field from the position and strength of discrete vortices in the flow field. The vorticity then follows by taking the curl of the induced velocity. In general the UAVM is able to capture the major flow structures around the wing: the LEV during the mid-stroke as well TEV are well defined. However, the LEV is over-predicted in UAVM, partly due to the assumption of constant shedding of the vortices at the leading edge. Moreover, the viscous diffusion, externally modeled into the UAVM, is less than in the NS computation or in the experiments. Finally, there is no mechanism present in UAVM that prevents the vortices in the flow field to pass through the wing. These three reasons may be the potential sources for the large difference in the forces during the first part of the stroke as depicted in Figure 7 between $t^{*}=0.05-0.15$ for HM1 and sine motion. The vorticity field at $t^{*}$ indeed shows presence of additional vortices upstream of the wing for the UAVM results that are not present in the PIV measurements and NS computations. Note also small blob of vorticity on the suction side of the wing near the TE at $t^{*}=0.1$ in UAVM that seems to be connected to the vorticity beneath the pressure side of the wing. On the other hand, HM2, the vorticity field obtained from UAVM $\left(t^{*}=0.1\right)$ is closer to that of the PIV and NS computation. This is also consistent with the better correlation of the forces around this time instant for HM2. Exact quantification of these artificial effects requires future study. 


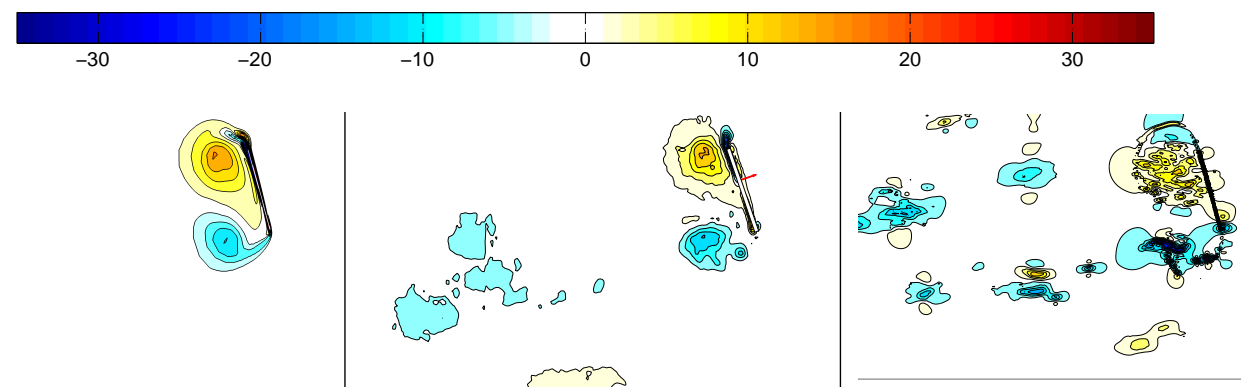

(a) $t^{*}=0.0$
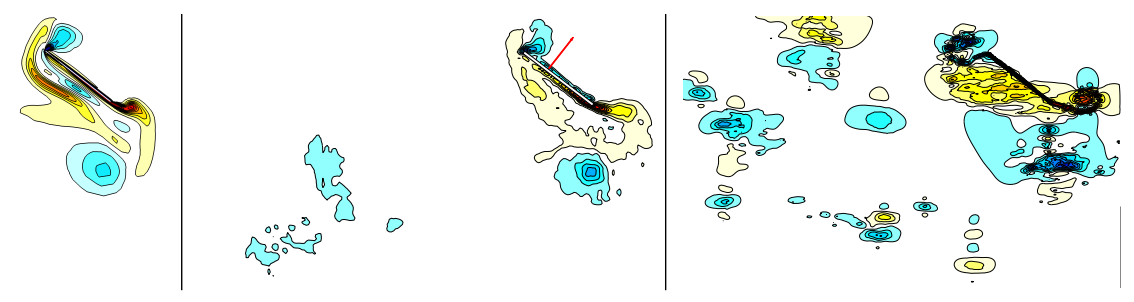

(b) $t^{*}=0.1$
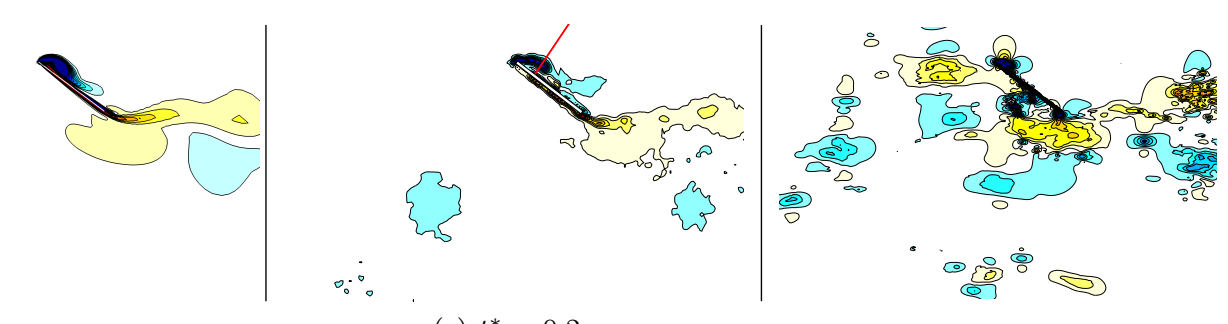

(c) $t^{*}=0.2$
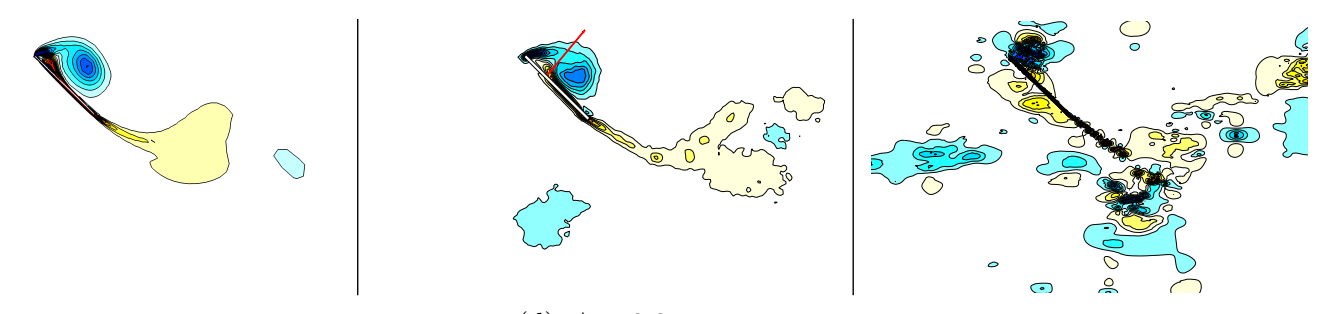

(d) $t^{*}=0.3$
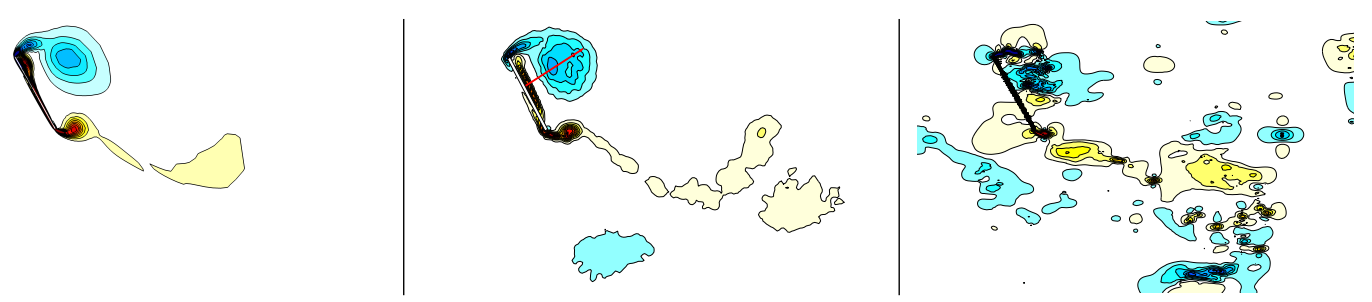

(e) $t^{*}=0.4$
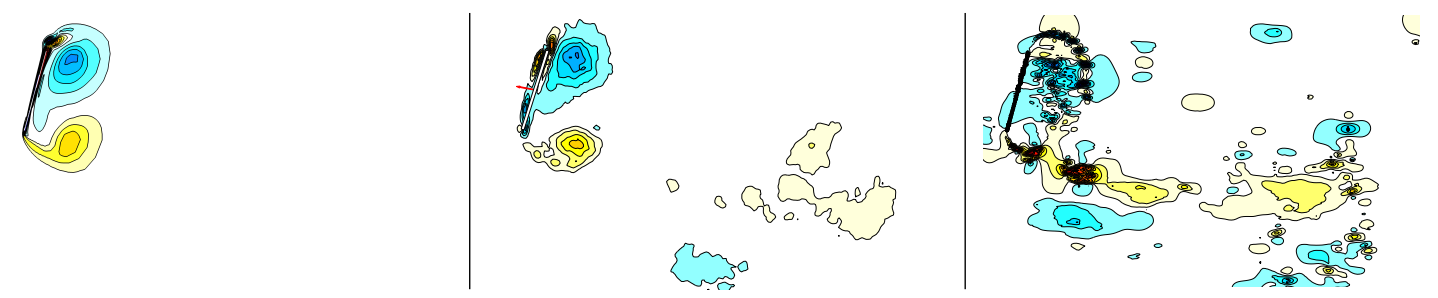

(f) $t^{*}=0.5$

Figure 8: Comparison of vorticity plots for HM1 base at half span, left image is the NS computation result, the middle image is the PIV experiment, and the right image displays the vorticity calculated from the unsteady aerodynamic vortex model 


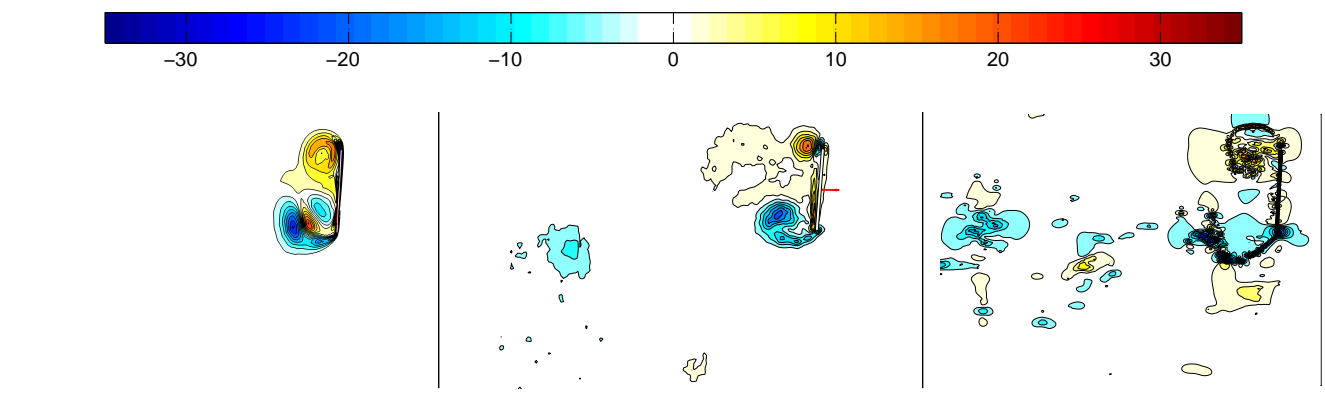

(a) $t^{*}=0.0$
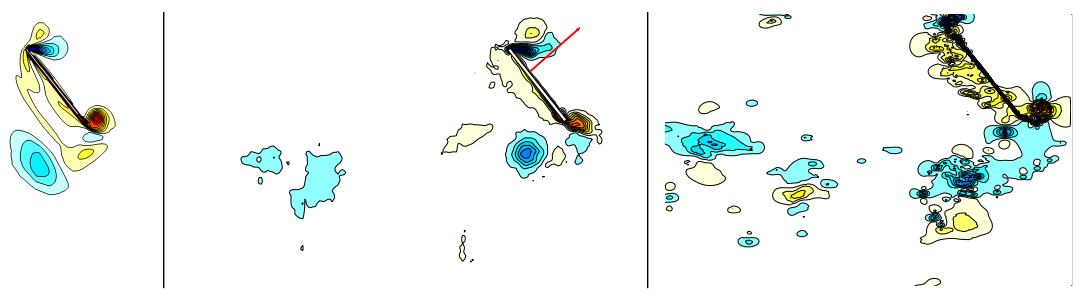

(b) $t^{*}=0.1$

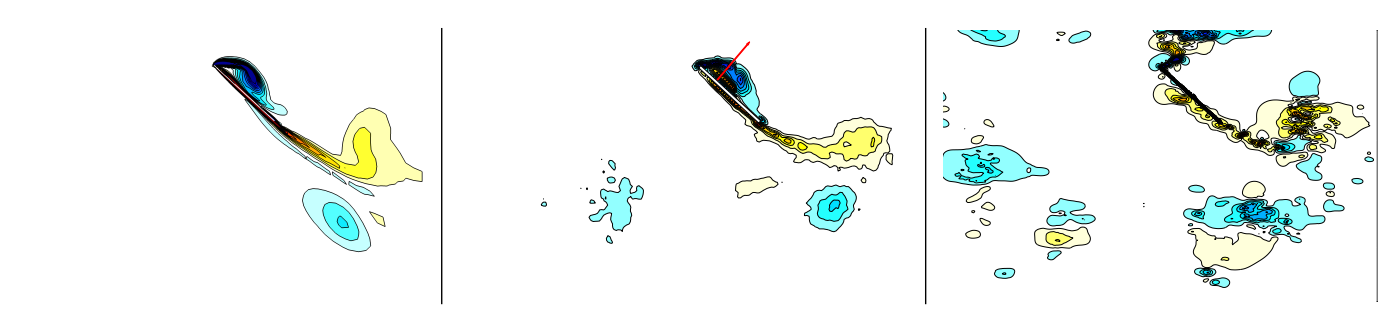

(c) $t^{*}=0.2$
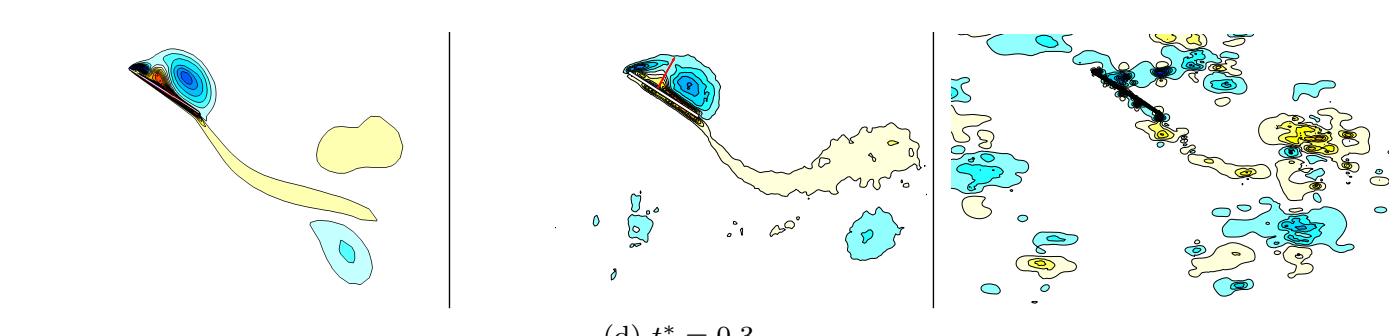

(d) $t^{*}=0.3$
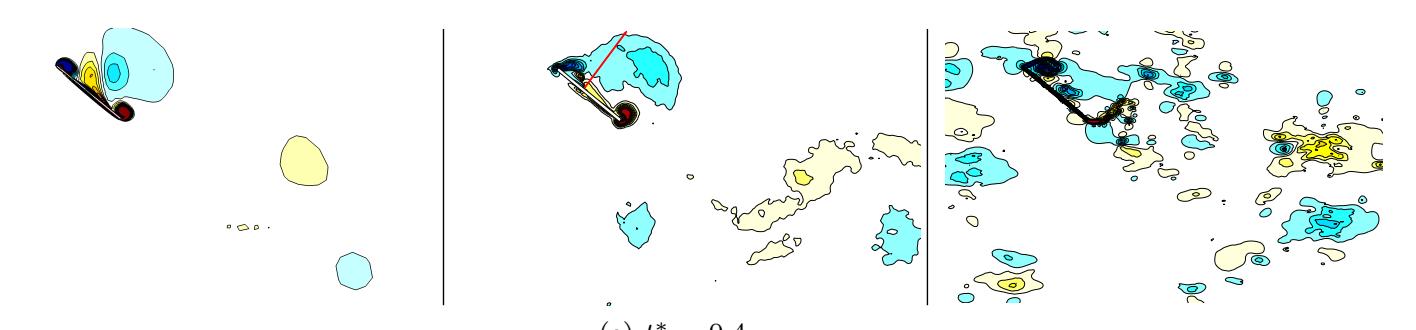

(e) $t^{*}=0.4$
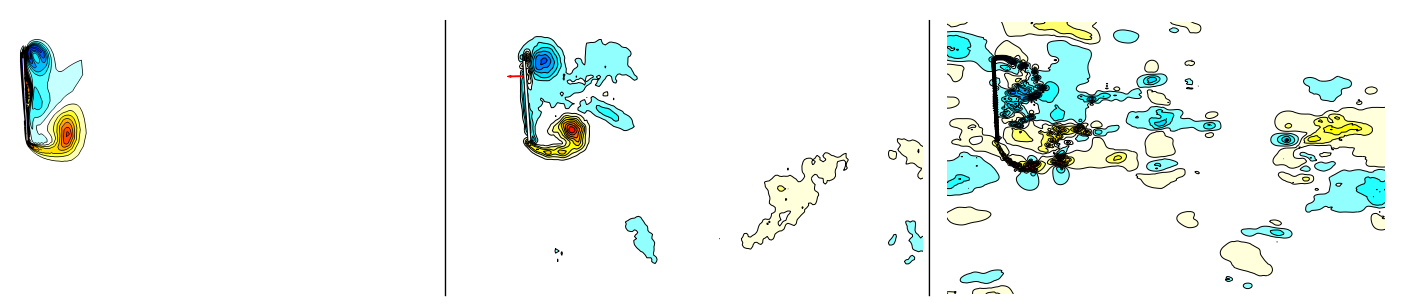

(f) $t^{*}=0.5$

Figure 9: Comparison of vorticity plots for HM2 base at half span, left image is the NS computation result, the middle image is the PIV experiment, and the right image displays the vorticity calculated from the unsteady aerodynamic vortex model 


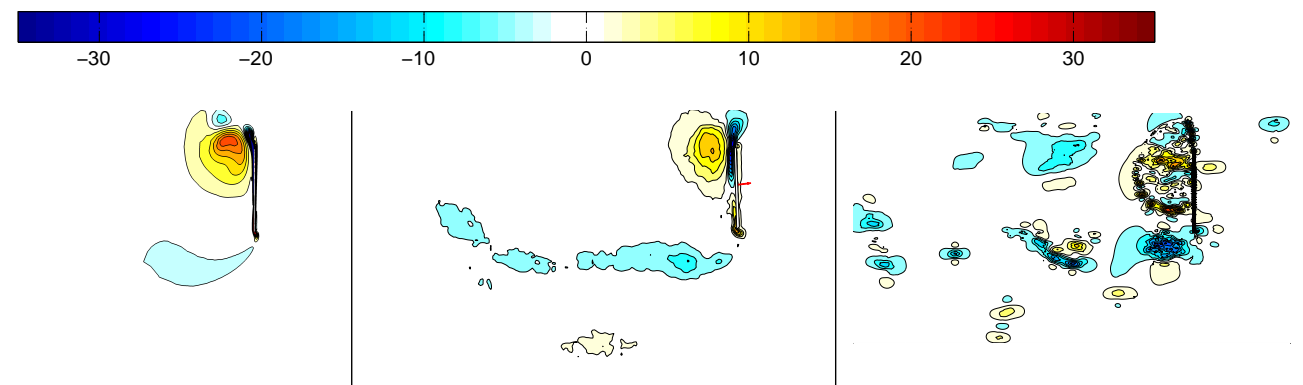

(a) $t^{*}=0.0$
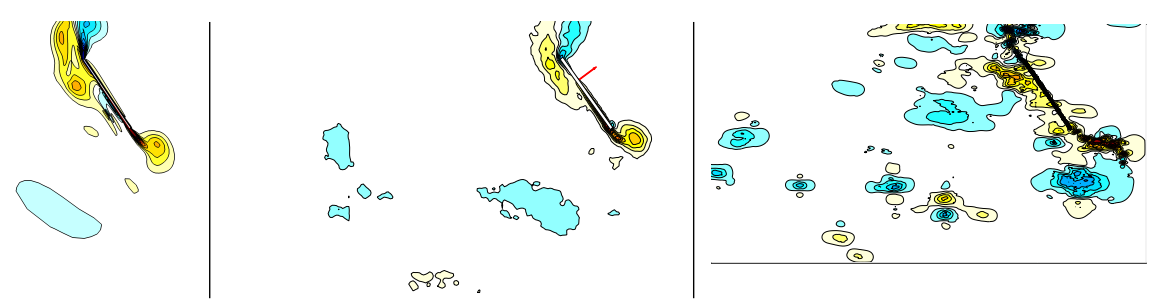

(b) $t^{*}=0.1$
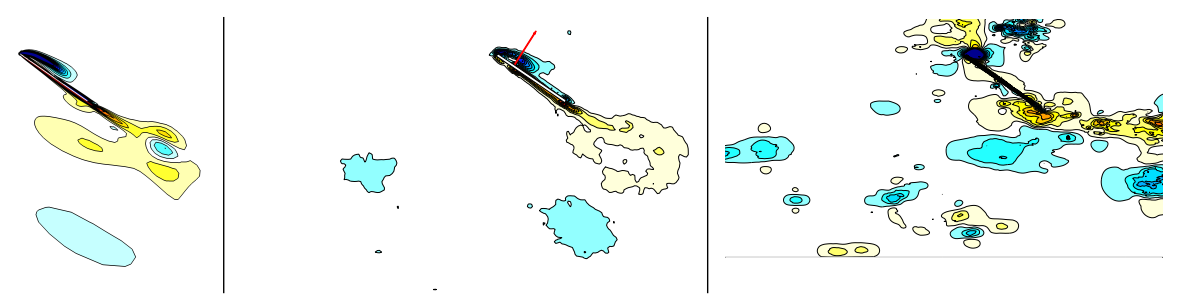

(c) $t^{*}=0.2$
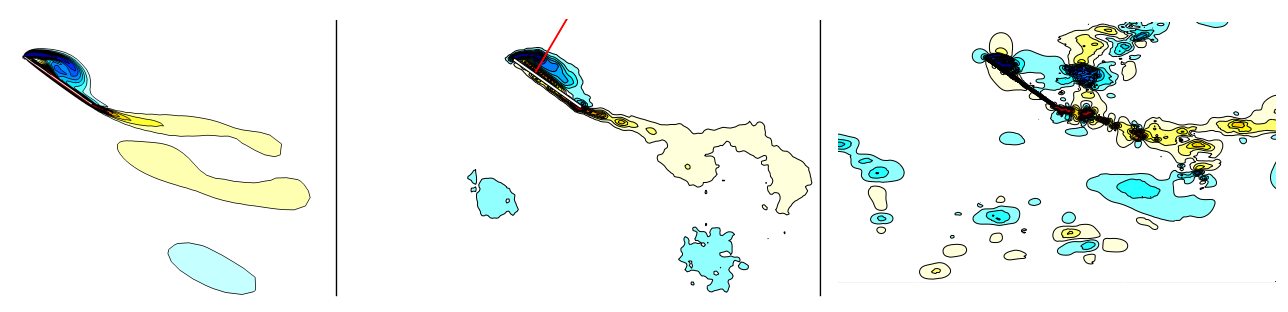

(d) $t^{*}=0.3$
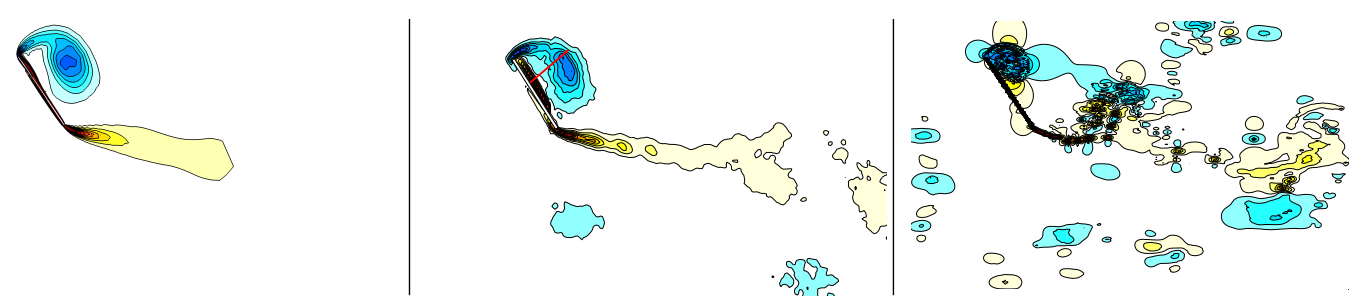

(e) $t^{*}=0.4$
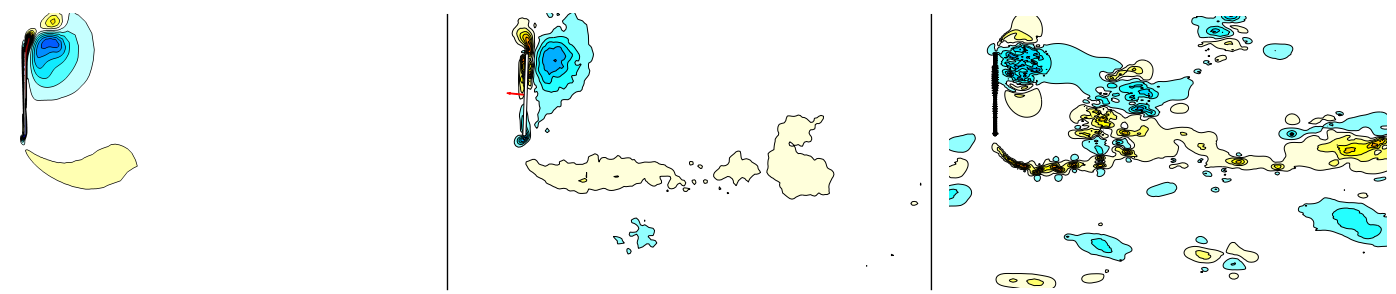

(f) $t^{*}=0.5$

Figure 10: Comparison of vorticity plots for Sine base at half span, left image is the NS computation result, the middle image is the PIV experiment, and the right image displays the vorticity calculated from the unsteady aerodynamic vortex model 


\section{Conclusions}

This paper assesses the force generation and the flow field of a hovering three-dimensional Zimmerman wing undergoing bio-inspired kinematics obtained using direct force and Particle Image Velocimetry measurements, Navier-Stokes equation solver, and Unsteady Aerodynamic Vortex Model. Three pitch-plunge motions derived from hawkmoth kinematics are considered, namely: pitch angle equal to i) feathering angle without elevation angle (HM1); ii) feathering angle with a correction for the elevation angle (HM2); and iii) a sinusoidal fit of the feathering angle (sine).

Aerodynamic forces calculated by solving the Navier-Stokes equations are consistent with the direct force measurements. The time-averaged forces are lower by 82 to $87 \%$ of the force coefficients measured experimentally. The small differences can be related to a number of factors. First, the presence of wall and blockage effects in the water channel might increase the forces measured in the experiment. Second,the presence of an induced flow, which may not be well-developed in the simulations may also result in higher force coefficients. Third, the Navier-Stokes equation solutions are computed by assuming laminar flow, therefore turbulence effects or transitional effects from laminar-to-turbulence are not included but may be present in the experiments. Similar to the forces, the vorticity field computed from Navier-Stokes equation solver shows qualitatively similar flow structures. The vorticity magnitude is slightly smaller than what has been observed in the PIV measurements. The computational time for five motion cycles was around 16 hours on 24 CPUs.

The Unsteady Aerodynamic Vortex Model captures the major trends of the force history with larger differences in the amplitude and phase than the Navier-Stokes computations. The computational time for five motion cycles was around six hours on 12 CPUs. For the three cases considered, the Unsteady Aerodynamic Vortex Model under-predicts the thrust in the first half of the motion stroke, while the thrust in the second half is much greater. The resulting vorticity field illustrates that the strength of the leading-edge vortex is over-predicted, which in turn affects the wing-wake interaction that occurs right after the stroke reversal. Further potential sources of this discrepancy are the simplified model of vortex diffusion and the artificial wing-wake interaction. As a result the time-averaged thrust coefficient from the Unsteady Aerodynamic Vortex Model yielded closer values than the Navier-Stokes equations for the force measurements due to cancellation effects for HM1 and sine motions, while the difference was greater for HM2 that showed the best agreement with the experiment and the Navier-Stokes computations, where the vorticity field showed the least wing-wake interaction. The standard deviation is consistently greater than that of the Navier-Stokes computations.

\section{Acknowledgments}

This research was sponsored in part by the Air Force Office of Scientific Researchs Multidisciplinary University Research Initiative (MURI) contract number FA9550-07-1-0547.

\section{References}

\footnotetext{
${ }^{1}$ Willmott, A. P. and Ellingtion, C. P., "The Mechanics of Flight in the Hawkmoth Manduca Sexta," The Journal of Experimental Biology, Vol. 200, 1997, pp. 2705-2722.

${ }^{2}$ Miller, L. A. and Peskin, C. S., "A Computational Fluid Dynamics of 'Clap and Fling' in the Smallest Insects," The Journal of Experimental Biology, Vol. 208, 2005, pp. 195-212.

${ }^{3}$ Shyy, W., Lian, Y., Tang, J., Viieru, D., and Liu, H., Aerodynamics of Low Reynolds Number Flyers, Cambridge University Press, 2008.

${ }^{4}$ Trizila, P., Kang, C., Aono, H., Shyy, W., and Visbal, M., "Low-Reynolds-Number Aerodynamics of a Flapping Rigid Flat Plate," AIAA Journal, Vol. 49, No. 4, April 2011, pp. 806-823.

${ }^{5} \mathrm{Ol}$, M., Bernal, L. P., Kang, C., and Shyy, W., "Shallow and Deep Dynamic Stall for Flapping Low Reynolds Number Airfoils," Experiments in Fluids, Vol. 46, No. 5, 2009, pp. 883-901.

${ }^{6}$ Kang, C., Aono, H., Baik, Y., Bernal, L., and Shyy, W., "Fluid Dynamics of Pitching and Plunging Flat Plate at Intermediate Reynolds Numbers," AIAA Journal, in press.

${ }^{7}$ Vandenheede, R. B. R., Bernal, L. P., Morrison, C. L., and Humbert, S., "Force Generation of Bio-inspired Hover Kinematics," AIAA 2012-0708, 2012.

${ }^{8}$ Gogulapati, A. and Friedmann, P., "Approximate Aerodynamic and Aeroelastic Modeling of Flapping Wings in Hover and Forward Flight," 52 $2^{\text {nd }}$ AIAA/ASME/ASCE/AHS/ASC Structures, Structural Dynamics, and Materials Conference, Vol. AIAA 2011-2008, April 2011.
} 
${ }^{9}$ Shyy, W., Aono, H., Chimakurthi, S., Trizila, P., Kang, C., Cesnik, C., and Liu, H., "Recent Progress in Flapping Wing Aerodynamics and Aeroelasticity," Progress in Aerospace Sciences, Vol. 46, 2010, pp. 284-327.

${ }^{10}$ Ramananarivo, S., Godoy-Diana, R., and Thiria, B., "Rather than Resonance, Flapping Wing Flyers may Play on Aerodynamics to improve Performance," Proceedings of the National Academy of Sciences, Vol. 108, No. 15, 2011, pp. 5964.

${ }^{11}$ Kang, C., Aono, H., Cesnik, C., and Shyy, W., "Effects of Flexibility on the Aerodynamic Performance of Flapping Wings," Journal of Fluid Mechanics, Vol. 689, 2011, pp. 32-74.

${ }^{12}$ Liu, H., Ellington, C. P., Kawachi, K., van den Berg, C., and Wellmott, A. P., "A Computational Fluid Dynamic Study of Hawkmoth Hovering," The Journal of Experimental Biology, Vol. 201, 1998, pp. 461-477.

${ }^{13}$ Ansari, S. A., Zbikowski, R., and Knowles, K., "Non-linear Unsteady Aerodynamic Model for Insect-like Flapping Wing in Hover. Part 1: Methodology and Analysis," Proceedings of the I MECH E Part G Journal of Aerospace Engineering, Vol. 220, No. 2, 2006, pp. 61-83.

${ }^{14}$ Ansari, S. A., Zbikowski, R., and Knowles, K., "Non-linear Unsteady Aerodynamic Model for Insect-like Flapping Wing in Hover. Part 2: implimentation and Validation," Proceedings of the I MECH E Part G Journal of Aerospace Engineering, Vol. 220, No. 2, 2006, pp. 169-186.

${ }^{15}$ Gogulapati, A., Friedmann, P., and Shyy, W., "Non-linear Aeroelastic Effects in Flapping Wing Micro Air Vehicles," $49^{\text {th }}$ AIAA/ASME/ASCE/AHS/ASC Structures, Structural Dynamics, and Materials conference, Vol. AIAA 2008-1817, April 2008.

${ }^{16}$ Gogulapati, A., Friedmann, P., and Shyy, W., "Approximate Aeroelastic Analysis of Flapping Wings in Hover," International Forum for Aeroelasticity and Structural Dynamics, Vol. IFASD 2009-143, June 2009.

${ }^{17}$ Balay, S., Gropp, W. D., McInnes, L., and Smith, B., "Efficient managment of parallelism in object oriented numerical software libraries," Modern Software Tools in Scientific Computing, 1997, pp. 163-202.

${ }^{18}$ Balay, S., PETSc Users Manual, Argonne National Laboratory, 2010.

${ }^{19}$ Balay, S., Brown, J., Buschelman, K., Gropp, W. D., Kaushik, D., Knepley, M. G., McInnes, L. C., Smith, B. F., and Zhang, H., "PETSc Web page," 2011.

${ }^{20}$ Flagout, R., Yang, U., Sloot, P., Hoekstra, A., Tan, C., and Dongarra, J., "Hypre: A Library of High Performance Preconditioners," Computational Science - ICCS 2002, 2002, pp. 632-641.

${ }^{21}$ Luke, E. A. and George, T., "Loci: a Rule-based Framework for Parallel Multi-disciplinary Simulation Synthesis," Journal of Functional Programming, Vol. 15, 2005, pp. 477-502.

${ }^{22}$ Thomas, P. and Lombard, C., "Geometric Conservation Law and Application to Flow Computations on Moving Grids," AIAA Journal, Vol. 10, 1979, pp. 1030-1037.

${ }^{23}$ Kamakoti, R. and Shyy, W., "Evaluation of Geometric Conservation Law using Pressure-based Fluid Solver and Moving Grid Technique," International Journal of Heat and Fluid Flow, Vol. 7, 2004, pp. 851-865.

${ }^{24}$ Vandenheede, R. B. R., Force Generation of Bio-Inspired Hover Kinematics: An experimental campaign, Master's thesis, Delft University of Technology, June 2012.

${ }^{25}$ Shyy, W. and Liu, H., "Flapping Wings and Aerodynamic Lift: The Role of Leading-Edge Vortices," AIAA Journal, Vol. 45, No. 12, December 2007, pp. 2817-2819.

${ }^{26}$ Dickinson, M. H., Lehmann, F.-O., and Sane, S. P., "Wing Rotation and the Aerodynamic Basis of Insect Flight," Science, Vol. 284, 1999, pp. 1954-1960. 